\title{
Factors associated with postpartum hemorrhage maternal death in referral hospitals in Senegal and Mali: a cross-sectional epidemiological survey
}

\author{
Julie Tort ${ }^{1,2,3,8^{*}}$, Patrick Rozenberg ${ }^{4,5}$, Mamadou Traoré ${ }^{6}$, Pierre Fournier ${ }^{7}$ and Alexandre Dumont ${ }^{1,2}$
}

\begin{abstract}
Background: Postpartum hemorrhage (PPH) is the leading cause of maternal mortality in Sub-Saharan-Africa (SSA). Although clinical guidelines treating PPH are available, their implementation remains a great challenge in resource poor settings. A better understanding of the factors associated with PPH maternal mortality is critical for preventing risk of hospital-based maternal death. The purpose of this study was thus to assess which factors contribute to maternal death occurring during PPH. The factors were as follows: women's characteristics, aspects of pregnancy and delivery; components of PPH management; and organizational characteristics of the referral hospitals in Senegal and Mali.
\end{abstract}

Methods: A cross-sectional survey nested in a cluster randomized trial (QUARITE trial) was carried out in 46 referral hospitals during the pre-intervention period from October 2007 to September 2008 in Senegal and Mali. Individual and hospital characteristics data were collected through standardized questionnaires. A multivariable logistic mixed model was used to identify the factors that were significantly associated with PPH maternal death.

Results: Among the 3,278 women who experienced PPH, 178 (5.4 \%) of them died before hospital discharge. The factors that were significantly associated with PPH maternal mortality were: age over 35 years (adjusted OR $=2.16$ [1.26-3.72]), living in Mali (adjusted $\mathrm{OR}=1.84$ [1.13-3.00]), residing outside the region location of the hospital (adjusted $\mathrm{OR}=2.43$ [1.29-4.56]), pre-existing chronic disease before pregnancy (adjusted $\mathrm{OR}=7.54$ [2.54-22.44]), prepartum severe anemia (adjusted $\mathrm{OR}=6.65$ [3.77-11.74]), forceps or vacuum delivery (adjusted $\mathrm{OR}=2.63$ [1.19-5.81]), birth weight greater than 4000 grs (adjusted $\mathrm{OR}=2.54$ [1.26-5.10]), transfusion (adjusted $\mathrm{OR}=2.17$ [1.53-3.09]), transfer to another hospital (adjusted $\mathrm{OR}=13.35$ [6.20-28.76]). There was a smaller risk of PPH maternal death in hospitals with gynecologist-obstetrician (adjusted $\mathrm{OR}=0.55$ [0.35-0.89]) than those with only a general practitioner trained in emergency obstetric care (EmOC).

Conclusions: Our findings may have direct implications for preventing PPH maternal death in resource poor settings. In particular, we suggest anemia should be diagnosed and treated before delivery and inter-hospital transfer of women should be improved, as well as the management of blood banks for a quicker access to transfusion. Finally, an extent training of general practitioners in EmOC would contribute to the decrease of PPH maternal mortality.

Keywords: Postpartum hemorrhage, maternal mortality, Sub-Saharan Africa

\footnotetext{
* Correspondence: julie.tort@gmail.com

${ }^{1}$ Research Institute for Development, Paris Descartes University, Sorbonne

Paris Cité, MERIT - UMR 216, Paris, France

UPMC University, Paris, France

Full list of author information is available at the end of the article
}

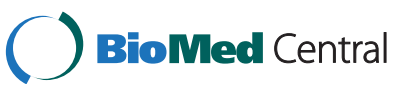

(c) 2015 Tort et al. Open Access This article is distributed under the terms of the Creative Commons Attribution 4.0 International License (http://creativecommons.org/licenses/by/4.0/), which permits unrestricted use, distribution, and reproduction in any medium, provided you give appropriate credit to the original author(s) and the source, provide a link to the Creative Commons license, and indicate if changes were made. The Creative Commons Public Domain Dedication waiver (http://creativecommons.org/publicdomain/zero/1.0/) applies to the data made available in this article, unless otherwise stated. 


\section{Background}

Maternal mortality remains a major health problem worldwide, particularly in sub-Saharan Africa (SSA) where more than $50 \%$ of maternal deaths occurred and where the lifetime risk of maternal death is 10 times higher than that in high-income countries [1]. Obstetric hemorrhage is the leading cause of maternal death in this region [2]. Most hemorrhages occur during the postpartum period [3] and are generally related to uterine atony, retained placenta and/or genital lacerations [4]. Clinical guidelines to treat post-partum hemorrhage (PPH) are available [5], but their implementation remains a great issue in resource poor settings.

There are three delays occurring during the diagnosis and management periods of PPH that are generally associated with a greater incidence in maternal mortality: delay in deciding to seek care (delay 1), delay in reaching the health facility (delay 2), delay in receiving quality emergency obstetric care (delay 3) [6]. Delay 3 is known to contribute more to maternal mortality than do delays 1 and 2 in SSA [6]. Late diagnoses, lack of drugs and supplies, referral delays, inadequate care or severe mismanagement and/or limited blood supply for transfusion are the most common problems observed [7] during the delay 3 in SSA [8].

In addition to this, the general maternal health status exerts a strong influence on the severity of PPH. For example, women presenting anemia, which is highly prevalent in SSA [9], are at better risk for severe PPH than others.

A better understanding of the factors associated with maternal death during PPH would help health care providers to rapidly identify women at highest risk for dying and provide appropriate care. To our knowledge, no previous studies have specifically addressed this question. Our objective was thus to identify the factors associated with maternal death occurring during $\mathrm{PPH}$ in referral hospitals in Senegal and Mali. The factors we tested were as follows: women's characteristics, aspects of pregnancy and delivery; PPH management; and organizational characteristics in maternity units,

\section{Methods}

\section{Setting}

This is a cross-sectional study nested in the one-year preintervention period of a multicentre cluster-randomized trial (QUARITE trial). The objective of this trial was to evaluate the effectiveness of a multifaceted educational intervention for reducing hospital-based maternal mortality in Senegal and Mali.

46 public referral hospitals (22 in Mali and 24 in Senegal) with more than 800 deliveries a year and a functional operating room were recruited from Sept 1, 2007, to Oct 30, 2011. The trial consisted of a 1-year pre-intervention or baseline period, a 2-year intervention period, and a 1-year post-intervention period.

The study protocol has already been published $[10,11]$. QUARITE trial is registered with ClinicalTrials.gov, number ISRCTN46950658. Ethical approval for QUARITE and this sub-study was granted by the ethics committee of Sainte-Justine Hospital in Montreal, Canada, and by the national ethics committees in Senegal and in Mali. The participating hospitals were included on the basis of informed consent by the local authorities. Collection of clinical data from hospital registers and medical records is authorized by the hospital authorities and does not require patient consent [11].

The 46 participant hospitals were representative of the existing public health referral system in both countries. The public health referral system, which is almost the only provider of modern health-care services in both countries, is based on district hospitals, regional hospitals, and national or teaching hospitals. These hospitals offer comprehensive emergency obstetric care in theory [12]. However, transfusion could be sometimes unavailable due to lack of blood products.

$\mathrm{PPH}$ cases are managed in the units where the complications arose. In district hospitals, the patients who require more specialized health care services (i.e. intensive care unit) are usually transferred to regional or national hospitals.

The intervention was not implemented during the baseline period of the trial. Therefore, there were no specific guidelines implemented by the research team during this period.

The participant hospitals represented $94 \%$ of all referral hospitals in Mali and Senegal and covered approximately $10 \%$ of all deliveries in both countries. According to available statistics in Mali and Senegal, among the remaining $90 \%$ of deliveries, approximately $50 \%$ occurred at home and $50 \%$ in community health care centers in Mali and approximately $68 \%$ occurred at home and $32 \%$ in community health care centers in Senegal $[13,14]$.

\section{Population}

All women who delivered in the participant hospitals during the study period were enrolled in the QUARITE trial.

In the present study, we only included women with $\mathrm{PPH}$ who delivered in participating hospitals during the baseline period (year 1, from October 2007 to October 2008). PPH was clinically assessed by the caregivers according to the visual estimation of excessive blood loss and patient status. The data collector recorded PPH only if the clinicians noted it in the clinical file. Women with uterine rupture or ante-partum hemorrhage (abruptio placentae or placenta praevia) or who died before delivery were excluded. 


\section{Data collection}

Characteristics of women, pregnancy, labor and delivery and $\mathrm{PPH}$ management were extracted from hospital registers and medical records and were registered every day by local trained data collectors (nurses or midwives) in a standard form. The standard form included a list of diagnoses related to the most frequent pathologies during pregnancy and delivery in Senegal and Mali and defined according to the Tenth International Classification of Diseases.

Hospital-based maternal deaths were identified among all the female deaths that occurred in the facility using the various registers available: admissions, hospitalizations (maternity and other services), operating rooms, and morgue. The cause of death was assessed by a doctor.

The quality and the completeness of the data were regularly controlled by the trial coordinator [11]. Missing data accounted for less than $1 \%$ of cases. Available resources for each hospital were registered at inclusion by the trial coordinator using the standard inventory developed by WHO for the global survey for monitoring maternal and perinatal health [15]. This measure considers organizational data at the facility level and no data were missing.

\section{Study variables}

The outcome was the hospital-based maternal death, measured as the vital status of the mother at hospital discharge categorized as alive or dead. All women transferred in another hospital after delivery were tracked by the data collectors or the national coordinator. The outcome was assessed in each case.

Four categories of potential risk factors for PPH maternal mortality were considered: characteristics of the women, and aspects of pregnancy and delivery before $\mathrm{PPH}$; components of PPH management; and organizational characteristics of the hospitals. Well known individual risk factors were selected from relevant literature [16-19]. Institutional or contextual factors were selected from information based on our field experience and inputs from health providers in participating hospitals.

The maternal preexisting characteristics were as follows: country of residence (Mali or Senegal); location of the woman's residence relative to the hospital in three categories: residence in the city of the hospital, outside the city of the hospital but in the same region, outside the region of the hospital; maternal age at delivery in three categories: $<20,20-35$ and $>35$ years.

Aspects of pregnancy, labor and delivery include: parity and previous cesarean delivery categorized as: nulliparous, multiparous without cesarean delivery, multiparous with cesarean delivery (one or more); number of prenatal visits categorized as: $0,1-4,>4$; multiple pregnancy; preexisting diseases before pregnancy including: positive serology of human immunodeficiency virus (HIV), chronic pulmonary, cardiac or renal diseases, sickle cell trait or chronic hypertension; prepartum severe anemia $(<7 \mathrm{~g} / \mathrm{dL})$; gestational hypertensive disorders including the following diagnoses: gestational hypertension, pre-eclamspia, eclampsia, hemolysis, elevated liver enzymes and low platelet count (HELLP) syndrome ; referral from another health facility; induction of labor; prolonged labor, including the following diagnoses: obstructed labor, cephalopelvic disproportion, dystocia, labor not progressing according to a normal partogram; mode of delivery categorized as: spontaneous vaginal delivery, C-section before the onset of labor, C-section after the onset of labor, forceps/vacuum extraction; and birth weight categorized as: $<2500$, 2500-4000, >4000 grs.

Three components of PPH management were analyzed: transfusion of blood products (fresh blood or red blood cells), hysterectomy and transfer after delivery to another hospital if the patient required more specialized health care services.

The organizational characteristics of the hospitals where the women gave birth included: the hospital type classified as hospital in the capital, regional hospital outside the capital or district hospital outside the capital; skilled staff for cardiopulmonary maternal resuscitation or hysterectomy; physician specialized in anesthesia; the qualification of the physician for obstetric care on staff categorized as: gynecologist-obstetrician, when a specialist is available in the hospital or general practitioner (GP) trained in emergency obstetric care (EmOC), when a trained GP is available but no gynecologist-obstetrician; availability of a blood bank, adult intensive care unit, peripartum and postpartum care guidelines or continuous medical training program.

\section{Statistical analysis}

Descriptive statistics were conducted to portray women's characteristics, aspects of pregnancy and delivery; PPH management; and organizational characteristics of hospitals. Then, the maternal mortality proportion was calculated overall and for each factor cited above.

To test the association between each factor and PPH maternal mortality, we used a series of random-intercept hierarchical logistic regression models to take into account the dependence of observations within hospitals.

A three-stage statistical procedure was used. First, a series of univariate hierarchical logistic regressions were performed to identify which factor was associated with PPH maternal mortality. The association was quantified with unadjusted odds ratios and their $95 \%$ confidence intervals. A critical p value inferior to $20 \%$ was chosen for variable selection in further analyses.

Second, a multivariate hierarchical logistic regression was performed using women's characteristics, aspects of 
pregnancy and delivery that were selected in the first step. A critical p value inferior to $5 \%$ was used for significance (model 1).

Third, a multivariate hierarchical logistic regression was performed using components of PPH management and controlling by the variables significant in model 1 . A critical $p$ value inferior to $5 \%$ was used for significance (model 2).

Finally, a multivariate hierarchical logistic regression was performed using organizational characteristics and controlling by the variables significant in model 2 (model 3 ).

The relevant interactions between any two variables were tested with the Wald test.

Cases with one or more missing values among the characteristics of women, delivery and PPH management were not included in the multivariate analyses $(n=49$ Women, $1,5 \%$ of total).

We estimated the relative contribution of characteristics of women, PPH management factors and institutional factors to the variability of maternal mortality between hospitals. To that purpose, we used the ratios of the random intercept variances [20].

The data were analyzed with Stata v.12 Software (Stata Corporation, College Station, TX).

\section{Results}

Among 84,924 women who delivered in participant hospitals and were included in the QUARITE trial during the pre-intervention period, 3,278 met the inclusion criteria (Fig. 1). Among these women with PPH, 178 $(5.4 \%)$ died in the postpartum period and before hospital discharge. These proportions varied considerably between hospitals from 0 to $23.1 \%$ (data not shown).

\section{Selection of women's characteristics, aspects of pregnancy and delivery}

The distribution of women's characteristics, aspects of pregnancy and delivery and their association with $\mathrm{PPH}$ maternal mortality are presented in Table 1. In multivariate analysis, age over 35 years, residence in Mali and outside the region where the hospital is located, preexisting diseases before pregnancy, severe chronic anemia, birth weight over 4000 grs and forceps or vacuum delivery were positively associated with risk of maternal death under PPH.

\section{Components of PPH management}

Transfusion was administered in $27.7 \%$ of women with $\mathrm{PPH}$ (Table 2). Some patients required a hysterectomy $(1.1 \%)$ or a transfer to another hospital $(1.1 \%)$. After adjustment for women's characteristics, aspects of pregnancy and delivery, the risk of death for women with PPH was 2.17 higher in women with transfusion, and 13.35 higher in women who required a transfer to another hospital. One-half of the transferred women died during transportation or after the admission to the other hospital. Hysterectomy was associated with an increased

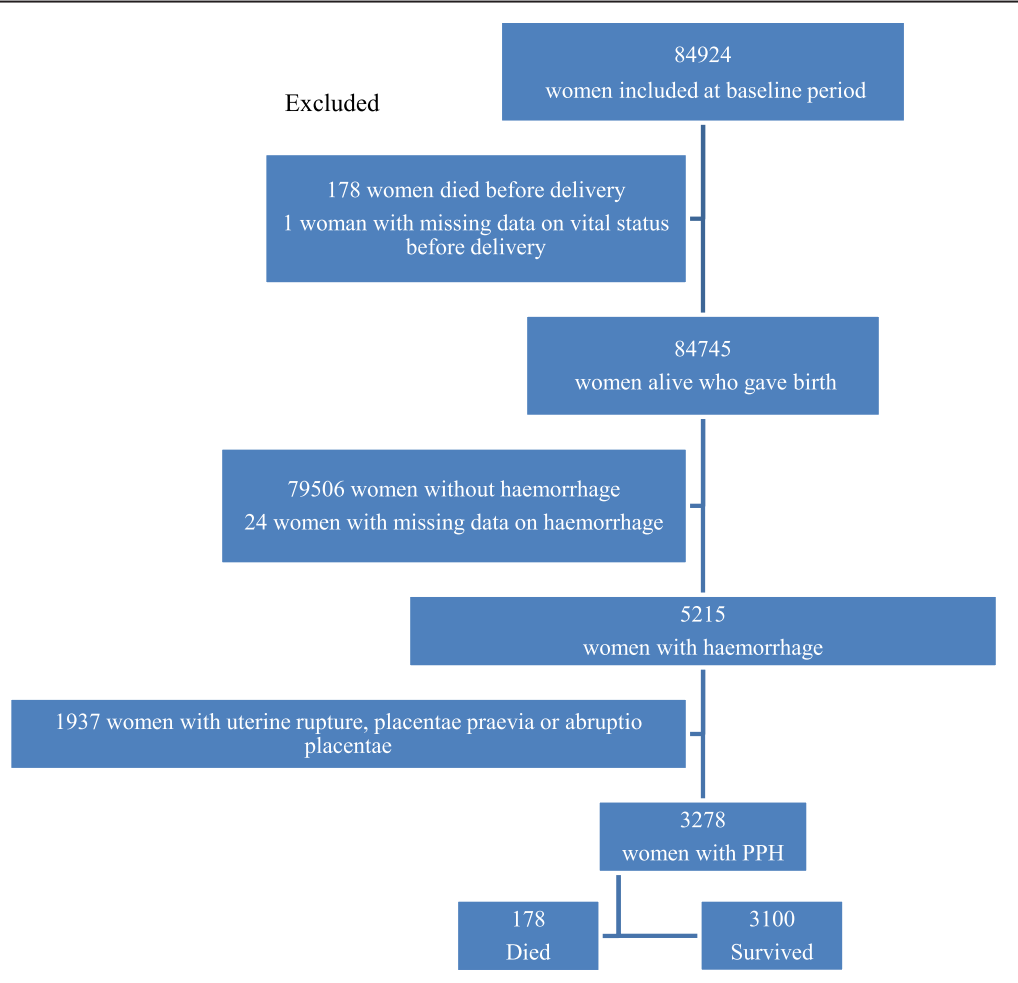

Fig. 1 Flow chart 
Table 1 Characteristics of women, pregnancy and delivery: distribution in the cohort of PPH and risk of death. Univariate and multivariate analysis

\begin{tabular}{|c|c|c|c|c|c|c|c|}
\hline & Number & Percent & CFR* (\%) & OR & $95 \% \mathrm{Cl}$ & $\mathrm{aOR}^{\mathrm{a}}$ & $95 \% \mathrm{Cl}$ \\
\hline \multicolumn{8}{|l|}{ Women and pregnancy } \\
\hline \multicolumn{8}{|l|}{ Country of residence } \\
\hline Senegal & 2551 & 77.8 & 4.8 & 1.00 & - & 1.00 & - \\
\hline Mali & 727 & 22.2 & 7.7 & 1.56 & $1.05-2.31$ & 1.84 & $1.13-3.00$ \\
\hline \multicolumn{8}{|l|}{ Location of residence } \\
\hline In the city of the hospital & 2058 & 63.5 & 5.0 & 1.00 & - & 1.00 & - \\
\hline Outside the city but in the same region & 982 & 30.3 & 5.1 & 1.18 & $0.77-1.80$ & 1.49 & $0.91-2.44$ \\
\hline Outside the region & 203 & 6.3 & 10.8 & 2.25 & $1.30-3.89$ & 2.43 & $1.29-4.56$ \\
\hline \multicolumn{8}{|l|}{ Age } \\
\hline Less than20 years & 649 & 19.8 & 4.2 & 1.00 & - & 1.00 & - \\
\hline Between 20 and 35 years & 2094 & 63.9 & 5.3 & 1.34 & $0.87-2.07$ & 1.48 & $0.93-2.44$ \\
\hline More than35 years & 535 & 16.3 & 7.5 & 1.88 & $1.13-3.13$ & 2.16 & $1.26-3.72$ \\
\hline \multicolumn{8}{|l|}{ Parity } \\
\hline Nulliparous & 691 & 21.1 & 5.5 & 1.00 & - & & \\
\hline Multiparous without previous caesarean & 2436 & 74.3 & 5.4 & 0.99 & $0.68-1.44$ & & \\
\hline Multiparous with previous caesarean & 151 & 4.6 & 8.0 & 0.90 & $0.41-1.98$ & & \\
\hline \multicolumn{8}{|l|}{ Number of prenatal visit } \\
\hline No visit & 510 & 15.8 & 7.5 & 1.47 & $0.99-2.17$ & 1.14 & $0.74-1.74$ \\
\hline Between 1 and 4 & 2514 & 78.0 & 4.8 & 1.00 & - & 1.00 & - \\
\hline More than4 & 201 & 6.2 & 6.5 & 1.38 & $0.76-2.52$ & 1.39 & $0.74-2.61$ \\
\hline Preexisting disease before pregnancy ${ }^{b}$ & 17 & 0.5 & 35.3 & 9.47 & $3.35-26.73$ & 7.54 & $2.54-22.44$ \\
\hline Multiple pregnancy & 184 & 5.6 & 5.4 & 1.00 & $0.52-1.96$ & & \\
\hline Prepartum severe anemia (<7 g/dL) & 85 & 2.6 & 25.9 & 7.05 & $4.15-11.98$ & 6.65 & $3.77-11.74$ \\
\hline Gestational hypertensive disorders $^{c}$ & 406 & 12.4 & 7.4 & 1.69 & $1.10-2.59$ & 1.57 & $0.98-2.52$ \\
\hline Referral from another health facility & 2045 & 62.4 & 5.9 & 1.32 & $0.95-1.84$ & 1.10 & $0.77-1.57$ \\
\hline \multicolumn{8}{|l|}{ Labor and delivery } \\
\hline Induction of labor & 554 & 16.9 & 4.3 & 0.84 & $0.53-1.35$ & & \\
\hline Prolonged labor & 710 & 21.7 & 7.8 & 1.64 & $1.15-2.33$ & 1.21 & $0.79-1.84$ \\
\hline \multicolumn{8}{|l|}{ Mode of delivery } \\
\hline Spontaneous vaginal & 2751 & 84.2 & 4.7 & 1.00 & - & 1.00 & - \\
\hline C-section after the onset of labor & 316 & 9.7 & 8.2 & 1.77 & $1.13-2.78$ & 1.54 & $0.91-2.61$ \\
\hline C-section before the onset of labor & 124 & 3.8 & 7.3 & 1.69 & $0.83-3.46$ & 1.86 & $0.89-3.91$ \\
\hline Forceps/Nacuum & 77 & 2.4 & 13.0 & 2.80 & $1.39-5.68$ & 2.63 & $1.19-5.81$ \\
\hline \multicolumn{8}{|l|}{ Birth weight } \\
\hline Less than 2500 grs & 1594 & 48.6 & 4.8 & 1.00 & - & 1.00 & - \\
\hline $2500-4000 \mathrm{gs}$ & 1600 & 48.8 & 5.3 & 1.09 & $0.79-1.50$ & 1.02 & $0.72-1.44$ \\
\hline More than 4000 grs & 84 & 2.6 & 20.2 & 4.88 & $2.69-8.84$ & 2.54 & $1.26-5.10$ \\
\hline Total & 3278 & 100.0 & 5.4 & & & & \\
\hline
\end{tabular}

*CFR: Case fatality rate. ${ }^{a}$ aOR: Odds Ratio and $95 \%$ confidence interval, adjusted for country of residence, location of residence, age, number of prenatal visit, preexisting disease before pregnancy, prepartum severe anemia, gestational hypertensive disorders, referred from another health facility, prolonged labor, mode of delivery and birth weight $(N=3179 \mathrm{PPH})$. Missing data: Location of residence $(n=35)$, number of prenatal visit $(n=53)$, induction of labor $(n=7)$, referred from

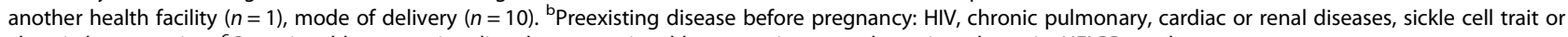
chronic hypertension. 'Gestational hypertensive disorders: gestational hypertension, preeclampsia, eclampsia, HELPP syndrome 
Table 2 Components of PPH management: distribution in the cohort of PPH and risk of death, univariate and multivariate analysis

\begin{tabular}{llcccccc}
\hline & Number & Percent & $\mathrm{CFR}^{*}(\%)$ & $\mathrm{OR}^{\mathrm{a}}$ & $95 \% \mathrm{Cl}$ & $\mathrm{aOR}$ & $95 \% \mathrm{Cl}$ \\
\hline Transfusion & 907 & 27.7 & 9.0 & 2.67 & $1.94-3.69$ & 2.17 & $1.53-3.09$ \\
Hysterectomy & 35 & 1.1 & 17.1 & 3.64 & $1.45-9.11$ & 2.18 & $0.79-6.02$ \\
Transfer to another hospital & 36 & 1.1 & 50.0 & 19.80 & $9.85-39.84$ & 13.35 & $6.20-28.76$ \\
\hline
\end{tabular}

${ }^{*}$ CFR: Case fatality rate. aOOR: Odds Ratio and $95 \%$ confidence interval, adjusted for country of residence, location of residence, age, preexisting disease before pregnancy, prepartum severe anemia, mode of delivery, birth weight, transfusion, hysterectomy and transfer to another hospital $(N=3227 \mathrm{PPH})$. Missing data: transfusion $(n=3)$, hysterectomy $(n=4)$, transfer to another hospital $(n=3)$

risk of case fatality, but the association was not significant when controlling for other factors.

\section{Organizational characteristics of hospitals}

The distribution of hospital characteristics is shown in Table 3, as well as their associations with PPH maternal death. More than half of women who experienced PPH were managed in regional hospitals, with a blood bank available, skilled staff for transfusion, cardiopulmonary maternal resuscitation and hysterectomy, and with guidelines and continuous medical training available. However, less than $50 \%$ of women were treated in hospitals with a physician specialized in anesthesia on staff and with an adult intensive care unit available. More than $80 \%$ of women were treated in a maternity unit with a gynecologist-obstetrician on staff.

In an univariate analysis, "gynecologist-obstetrician on staff" was significantly associated with a decrease risk of death $(p<0.05)$ (Table 3). After adjustment for all characteristics selected in the precedent models, this association remained significant. Hospitals having a gynecologistobstetrician were associated with a decreased risk of death by $45 \%(\mathrm{aOR}=0.55 ; 95 \% \mathrm{IC}:[0.35-0.89] ; p=0.014)$ when compared to hospitals that did not have.

Maternal characteristics, components of PPH management and organizational characteristics of hospitals that were included in the final model explained respectively 2.7, 1.4 and $10.9 \%$ of the between-hospital variability of maternal mortality ( $85 \%$ residual variability).

\section{Discussion}

To our knowledge, the present paper is the first to document the factors associated with $\mathrm{PPH}$ maternal death in referral hospitals in SSA. Our findings suggest that some women's characteristics, aspects of pregnancy and delivery; PPH management; and organizational characteristics

Table 3 Characteristics of the hospitals: distribution in the cohort of PPH and risk of death, univariate analysis and multivariate analysis

\begin{tabular}{|c|c|c|c|c|c|c|c|}
\hline & Number & Percent & CFR* $(\%)$ & OR & $95 \%$ IC & $\mathrm{aOR}^{\mathrm{a}}$ & $95 \%$ IC \\
\hline \multicolumn{8}{|l|}{ Type of hospital } \\
\hline Hospital in the Capital & 655 & 20,0 & 4.3 & 1.00 & - & & \\
\hline Regional hospital outside the capital & 1668 & 50.9 & 5.3 & 1.30 & $0.76-2.22$ & & \\
\hline District hospital outside the capital & 955 & 29.1 & 6.5 & 1.69 & $0.98-2.93$ & & \\
\hline \multicolumn{8}{|l|}{ Specialized care services availability } \\
\hline Blood bank & 1982 & 60.5 & 5.9 & 1.27 & $0.84-1.92$ & & \\
\hline Adult intensive care unit & 1516 & 46.2 & 5.5 & 1.07 & $0.71-1.63$ & & \\
\hline \multicolumn{8}{|l|}{ Human resource availability } \\
\hline Skilled staff for cardiopulmonary resuscitation & 1886 & 57.5 & 4.8 & 0.73 & $0.50-1.09$ & 0.98 & $0.65-1.47$ \\
\hline Skilled staff for hysterectomy & 3232 & 98.6 & 5.4 & 0.66 & $0.18-2.43$ & & \\
\hline \multicolumn{8}{|l|}{ Qualification of physician for obstetric care on staff } \\
\hline Trained general practitioner & 558 & 17.0 & 9.7 & 1.00 & - & 1.00 & - \\
\hline Gynecologist-obstetrician & 2720 & 83.0 & 6.1 & 0.45 & $0.32-0.62$ & 0.55 & $0.35-0.89$ \\
\hline Physician specialised in anaesthesia & 1323 & 40.4 & 4.5 & 0.69 & $0.45-1.05$ & 0.87 & $0.57-1.34$ \\
\hline \multicolumn{8}{|l|}{ Protocol and training } \\
\hline Intrapartum care guidelines & 2650 & 80.8 & 5.5 & 1.19 & $0.68-2.06$ & & \\
\hline Postpartum care guidelines & 2650 & 80.8 & 5.5 & 1.19 & $0.68-2.06$ & & \\
\hline Continuous medical training & 2562 & 78.2 & 5.5 & 1.12 & $0.67-1.87$ & & \\
\hline
\end{tabular}

*CFR: Case fatality rate. aOOR: Odds Ratio and $95 \%$ confidence interval, adjusted for country of residence, location of residence, age, pre-existing diseases before pregnancy, prepartum severe anemia, mode of delivery and birth weight; transfusion and transfer to another hospital; and for gynecologist-obstetrician, physician specialist in anesthesia, skilled staff for cardio-pulmonary resuscitation ( $N=3229 \mathrm{PPH})$. Type of hospital is not included because it is highly correlated with the qualification of physician for obstetric care on staff 
of hospitals are determinants of hospital-based PPH maternal death. In particular, these include the level of accessibility to the hospital, woman's health status before $\mathrm{PPH}$, the mode of delivery and birth weight. Availability of a gynecologist-obstetrician on staff is associated with a decreased risk of death, while transfusion and interhospital transfer are linked to an increased risk of death.

Our study showed that living in Mali is associated with a higher risk of PPH maternal mortality than in Senegal. No difference in the characteristics of women, pregnancy and childbirth between the two countries could explain this result. However, the prevalence of PPH in our study is higher in Senegal than in Mali. PPH definition might differ between the two countries. In Senegal, PPH diagnosis is mainly based on visual estimation of blood loss (i.e. estimation over $500 \mathrm{~mL}$ ), while in Mali, this is mainly based on visual estimation and patient status. The definition in Mali may lead to detect more severe $\mathrm{PPH}$ cases than in Senegal. This could explain why the case fatality rate is higher in Mali than in Senegal. In addition, the number of regional hospitals participating to the QUARITE Trial is higher in Senegal [11] than in Mali [4], while the number of district hospitals is higher in Mali [12] than in Senegal [7]. Health care professionals are globally more qualified in regional hospitals than in district hospitals, and they are able to diagnose PPH cases more likely in the higher level of care.

Our study showed that several women's characteristics, aspects of pregnancy and delivery are associated with PPH maternal mortality. This could help care providers in referral hospitals in Mali and Senegal to strengthen both diagnosis and treatment of PPH among women at high risk. Some characteristics are already known as risk factors for severe PPH: age over 35 years old, preexisting disease before the occurrence of $\mathrm{PPH}$ including severe anemia, instrumental delivery and birth weight over $4000 \mathrm{~g} \mathrm{[16-19]}$. Chronic diseases alter the physiological health status of the woman and increase the risk of organ dysfunctions when a hemorrhagic complication arises [21]. Moreover, a forceps or vacuum-based delivery or a birth weight greater than $4000 \mathrm{~g}$ increases the use of episiotomy or vaginal lacerations and thus more blood loss [22, 23]. From this arose the need of careful monitoring during immediate post-partum period.

The level of accessibility to referral hospitals is also a well-known risk factor for maternal death in SSA [6]. Long transport times between home and health care facility, increase the odd of poor health status of the women when admitted to the referral hospital. The inherent issues of communication existing between the health care facilities in SSA [6] also increase care delays and may lead to dramatic high case fatality rate $(50 \%$ in our study). In such a context, it is necessary to improve identification of women who require transfer, to ensure an efficient transfer organization and the quality of transportation. Particularly, the non-pneumatic antishock garment could make a significant contribution to help women survive delays due to transfer [24].

Paradoxically, our findings showed transfusion is associated with a higher risk of maternal death. Transfusion is however an essential care in the management of PPH. It helps to maintain appropriate blood pressure in case of significant blood loss. This association may thus reflect an indication bias because transfusion is more likely performed among women presenting the most severe $\mathrm{PPH}$. From this, we believe that a significant proportion of maternal deaths was probably related to severe $\mathrm{PPH}$ and that transfusion may have been performed too late or with insufficient quantity of blood products [8].

In our study, a gynecologist-obstetrician on staff is significantly associated with a decreased of risk of PPH maternal mortality. When a physician specialist is not available in the hospital, a trained general practitioner (GP) is in charge for the maternity unit. These results suggest that training in emergency obstetric care of GPs is probably insufficient. In both countries this training essentially allows to overcome the lack of obstetrician in the district hospitals. However, this training is informal and lasts over a few months. There is no examination or certification of achievement or degree. Knowledge of these physicians is thus limited and may not allow them to quickly diagnose PPH and provide quality care. This can also increase delays in decision-making and particularly the use of surgical treatments (arterial ligation, hysterectomy). Our findings confirm that the GPs are more likely employed in district hospitals than obstetricians, where a small annual amount of deliveries occur (data not shown), with less qualified care providers and limited drugs and supplies. The QUARITE trial showed that health care professionals training in emergency obstetric care had led to a significant reduction in maternal mortality in the district hospitals [10].

This study presents several strengths. Data were collected from 46 referral hospitals in Senegal and Mali, which were representative of the existing health system in both countries. Thus, this approach ensures the external validity of the study, whose findings could be easily generalized to health systems with similar characteristics. Sample size was large enough to perform hierarchical analyses with high statistical power $(N=3278$ women). Moreover, information on the vital status of women came from various sources, as a consequence, the number of hospitalbased maternal deaths was considered exhaustive.

However, our study also presents some limitations. First, blood loss was measured only with a subjective method by care givers and PPH may have been underestimated in our study [25]. The reported rate of PPH in our study was relatively small with $3.9 \%$. That rate was lower than that observed in African health care facilities as 
shown in Calvert et al. [25]. Second, it remains possible that diagnoses were under-reported in medical files. That bias may reduce the strength of the association between studied maternal characteristics and PPH case fatality. Third, we did not assess if the care providers were aware of the active management of the third stage of labor recommendations for PPH prevention and whether or not it was implemented. Fourth, a large part of the between-hospital variability in PPH maternal mortality remained unexplained. This heterogeneity may be due to differences in medical practices that were not measured in our study. Younger physicians working alone in rural settings may have different practices than older ones working in urban settings. Taking this information into account would improve the performance of our predictive model. Finally, most women in this study were discharged from the hospital two days or less after delivery. Maternal deaths occurring at home were not recorded. Data analyzed included information only from women who sought care at participant facilities, and excluded information on those delivered at home or referred from another health care facility after delivery. Therefore, maternal mortality in the population cannot be inferred and these results should be used only by clinicians to reduce hospital-based mortality related to $\mathrm{PPH}$.

\section{Conclusions}

Our findings may have direct implications for clinicians in resource poor settings. We demonstrated that women's characteristics, aspects of pregnancy and delivery; $\mathrm{PPH}$ management; and organizational characteristics of hospitals are risk factors for PPH maternal mortality in SSA. We suggest that severe antepartum anemia should be diagnosed more accurately and treated before delivery, while effort should be put on improving the capacity of the blood banks and making blood quickly accessible when needed. Early detection of PPH and timely decisionmaking for transfusion are critical. It is also important to improve the identification of women who need to be transferred to another facility and transportation system. Finally, our findings suggest that training in emergency obstetric care of GPs is probably insufficient. If that training may respond to the shortage of obstetricians, whose training is time and money consuming; on the other hand it prevents the same quality care. A better supervision and extensive training are essential keys to reduce gaps in knowledge and to prevent PPH maternal death.

\section{Competing interests}

The authors declare that they have no competing interests.

\section{Authors' contributions}

AD, MT and PF participated in developing the QUARITE trial. JT led the data analysis under the supervision of AD. JT and AD interpreted the results. The writing of the paper was led by JT under the supervision of AD and PR. All authors read and approval the final manuscript.
Authors' information

Not applicable.

Availability of data and materials

Not applicable.

\section{Acknowledgments}

We are grateful to all the women who participated in the trial. We thank all the medical and administrative staff of the 46 participating hospitals for their valuable contribution in this trial. We wish to thank the Canadian Institutes of Health Research (CIHR), which have fully funded the QUARITE trial and Paris Diderot University, which provided a salary grant to JT for this research.

\section{Author details}

${ }^{1}$ Research Institute for Development, Paris Descartes University, Sorbonne Paris Cité, MERIT - UMR 216, Paris, France. ${ }^{2}$ UPMC University, Paris, France. ${ }^{3}$ Paris Diderot University, Paris, France. ${ }^{4}$ Department of Obstetrics and Gynecology, Poissy Saint-Germain Hospital, Poissy, France. ${ }^{5}$ EA7285, Clinical risk and safety in women's health and perinatal health, University of Versailles Saint-Quentin (UVSQ), St Quentin en Yvelines, France. ${ }^{6}$ URFOSAME, Referral health center of the Commune V, Bamako, Mali. ${ }^{7}$ Research Centre of CHUM (CRCHUM), University of Montreal, Montreal, Canada. ${ }^{8}$ UMR 216, Faculté de Pharmacie, Laboratoire de Parasitologie, 4 Avenue de l'Observatoire, 75270 Paris Cedex 6, France.

Received: 13 November 2014 Accepted: 25 September 2015

Published online: 30 September 2015

\section{References}

1. WHO, UNICEF, UNFPA and The World Bank estmimates. Trends in maternal mortality: 1990 to 2010. Geneva: World Health Organization; 2012.

2. Say L, Chou D, Gemmill A, Tunçalp O, Moller A-B, Daniels J, et al. Global causes of maternal death: a WHO systematic analysis. Lancet Glob Health. 2014;2(6):e323-33.

3. Haeri S, Dildy 3rd GA. Maternal mortality from hemorrhage. Semin Perinatol. 2012;36(1):48-55.

4. Bateman BT, Berman MF, Riley LE, Leffert LR. The epidemiology of postpartum hemorrhage in a large, nationwide sample of deliveries. Anesth Analg. 2010;110(5):1368-73.

5. Tunçalp Ö, Souza JP, Gülmezoglu M. New WHO recommendations on prevention and treatment of postpartum hemorrhage. Int J Gynecol Obstet. 2013;123(3):254-6.

6. Knight HE, Self A, Kennedy SH. Why are women dying when they reach hospital on time? A systematic review of the «third delay ». PloS One. 2013;8(5), e63846.

7. Combs Thorsen V, Sundby J, Malata A. Piecing Together the Maternal Death Puzzle through Narratives: The Three Delays Model Revisited. PLoS ONE. 2012;7(12), e52090.

8. Bates I, Chapotera GK, McKew S, van den Broek N. Maternal mortality in sub-Saharan Africa: the contribution of ineffective blood transfusion services. BJOG Int J Obstet Gynaecol. 2008;115(11):1331-9.

9. Stevens GA, Finucane MM, De-Regil LM, Paciorek CJ, Flaxman SR, Branca F, et al. Global, regional, and national trends in haemoglobin concentration and prevalence of total and severe anaemia in children and pregnant and non-pregnant women for 1995-2011: a systematic analysis of populationrepresentative data. Lancet Glob Health. 2013;1 (1):e16-25.

10. Dumont A, Fournier P, Abrahamowicz M, Traoré M, Haddad S, Fraser WD. Quality of care, risk management, and technology in obstetrics to reduce hospital-based maternal mortality in Senegal and Mali (QUARITE): a cluster-randomised trial. The Lancet. 2013;382(9887):146-57.

11. Dumont A, Fournier P, Fraser W, Haddad S, Traore M, Diop I, et al. QUARITE (quality of care, risk management and technology in obstetrics): a clusterrandomized trial of a multifaceted intervention to improve emergency obstetric care in Senegal and Mali. Trials. 2009;10(1):85.

12. UNICEF, WHO, UNFPA. Guidelines for Monitoring the Availability and Use of Obstetric Services. Geneva: World Health Organization; 1997.

13. Agence Nationale de la Statistique et de la Démographie (ANSD) [Sénégal], ICF International. Enquête Démographique er de Santé Continue au Sénégal (EDS-Continue) 2012-2013. Calverton, Maryland, USA: ANSD et ICF International; 2012. 
14. Cellule de Planification et de Statistique (CPS/SSDSPF), Institut National de la statistique (INSTAT/MPATP, INFO-STAT, ICF International. Enquête Démographique et de la Santé au Mali 2012-2013. Rockville, Maryland, USA: CPS, INSTAT, INFO-STAT et ICF International; 2014.

15. Shah A, Faundes A, Machoki M, Bataglia V, Amokrane F, Donner A, et al. Methodological considerations in implementing the WHO Global Survey for Monitoring Maternal and Perinatal Health. Bull World Health Organ. 2008:86(2):126-31.

16. Al-Zirqi I, Vangen S, Forsen L, Stray-Pedersen B. Prevalence and risk factors of severe obstetric haemorrhage. BJOG Int J Obstet Gynaecol. 2008;115(10):1265-72.

17. Driessen M, Bouvier-Colle M-H, Dupont C, Khoshnood B, Rudigoz R-C, Deneux-Tharaux $C$, et al. Postpartum hemorrhage resulting from uterine atony after vaginal delivery: factors associated with severity. Obstet Gynecol. 2011;117(1):21-31.

18. Sheldon W, Blum J, Vogel J, Souza J, Gülmezoglu A, Winikoff B, et al. Postpartum haemorrhage management, risks, and maternal outcomes: findings from the World Health Organization Multicountry Survey on Maternal and Newborn Health. BJOG Int J Obstet Gynaecol. 2014;121 Suppl 1:5-13.

19. Kramer MS, Berg C, Abenhaim H, Dahhou M, Rouleau J, Mehrabadi A, et al, Incidence, risk factors, and temporal trends in severe postpartum hemorrhage. Am J Obstet Gynecol. 2013;209(5):449. e1-449.e7.

20. Merlo J, Chaix B, Ohlsson H, Beckman A, Johnell K, Hjerpe P, et al. A brief conceptual tutorial of multilevel analysis in social epidemiology: using measures of clustering in multilevel logistic regression to investigate contextual phenomena. J Epidemiol Community Health. 2006;60(4):290-7.

21. Souza JP, Gülmezoglu AM, Vogel J, Carroli G, Lumbiganon P, Qureshi Z, et al. Moving beyond essential interventions for reduction of maternal mortality (the WHO Multicountry Survey on Maternal and Newborn Health): a cross-sectional study. Lancet. 2013;381(9879):1747-55.

22. Jolly MC, Sebire NJ, Harris JP, Regan L, Robinson S. Risk factors for macrosomia and its clinical consequences: a study of 350,311 pregnancies. Eur J Obstet Gynecol Reprod Biol. 2003;111(1):9-14.

23. Fong A, Wu E, Pan D, Chung JH, Ogunyemi DA. Temporal trends and morbidities of vacuum, forceps, and combined use of both. J Matern Fetal Neonatal Med. 2014;1-6.

24. Turan J, Ojengbede O, Fathalla M, Mourad-Youssif M, Morhason-Bello IO, Nsima $\mathrm{D}$, et al. Positive effects of the non-pneumatic anti-shock garment on delays in accessing care for postpartum and postabortion hemorrhage in Egypt and Nigeria. J Womens Health 2002. 2011;20(1):91-8.

25. Calvert C, Thomas SL, Ronsmans C, Wagner KS, Adler AJ, Filippi V. Identifying regional variation in the prevalence of postpartum haemorrhage: a systematic review and meta-analysis. PloS One. 2012;7(7), e41114.

\section{Submit your next manuscript to BioMed Central and take full advantage of:}

- Convenient online submission

- Thorough peer review

- No space constraints or color figure charges

- Immediate publication on acceptance

- Inclusion in PubMed, CAS, Scopus and Google Scholar

- Research which is freely available for redistribution 